\title{
A plasmon-induced current loop in gold semi-shells
}

Michael Cortie* and Mike Ford.

Institute for Nanoscale Technology, University of Technology Sydney,

PO Box 123, Broadway, NSW 2007, Australia

* Prof. Michael Cortie, Tel : +61-2-9514-2208, Email : Michael.cortie@uts.edu.au,

Short title : Plasmonic current loop in gold semi-shells 
Abstract We perform a computational investigation of the optical properties of nanoscale gold 'semishells' and show how additional plasmon resonances develop as the shape is successively mutated from 'nanoshell' to 'nano-cup', 'half shell' and finally to 'nano-cap'. The effects of aspect ratio, surface roughness and cut-off height are explored. Of special interest is a new longitudinal resonance that generates an electric current loop. We predict that this will induce an orthogonal magnetic component that will sum with the magnetic component of incident light at certain orientations. Exploitation of this phenomenon in an ordered array of semi-shells may produce anomalous optical effects due to an altered magnetic permeability.

PAC Codes : $52.25 . \mathrm{Tx}, 73.20 \mathrm{Mf}$

\section{Introduction}

The optical properties of coinage metal $(\mathrm{Cu}, \mathrm{Ag}$, and $\mathrm{Au})$ nanoparticles are interesting as they exhibit strong plasmon resonances, usually in the visible part of the spectrum. As a result, suspensions or coatings of these particles may be quite strongly coloured. This has led to decorative applications $[1,2]$, but the phenomenon can also be exploited in fields as diverse as medical diagnostics [3], solar glazing $[4,5]$, resonant fluorescence enhancement [6], medical treatment [7-9], optical sensing [10], and spectrally or angularly selective filters [11]. A variety of shapes such as nanoshells, nanorods, and nanotriangles have been successfully synthesized, most commonly from gold or silver [12]. Core-shell nanoparticles of the metal-on-dielectric type ('nanoshells' [13]) are attractive on account of their optical properties which can be readily tuned by varying the ratio of inner and outer radii $\left(r_{\mathrm{i}} / r_{\mathrm{o}}\right)$ of the particle [14-18]. Reduction in the symmetry of these particles by somehow excising a part of the nanoshell generates a series of shapes we have termed 'semi-shells' [19]. Semi-shells encompass 'nano-caps' of low symmetry [20], through to 'nanocaps' with rotational symmetry [21], 'half-shells' and half-shell arrays [22-25], and 'nano-cups' [21] (figure 1). We consider semi-shells to be interesting for at least two reasons : their geometry offers an additional means (besides $r_{i} / r_{\mathrm{o}}$ ) to tune optical properties, and they can be prepared by physical or chemical deposition onto a polymer particle template [20-24, 26]. Previous work on the plasmon resonances in semi-shells has confirmed that the optical properties are also influenced by geometric asymmetry and by the orientation of the particle with respect to the light, as well as the fact that these shapes can sustain quadrupole resonances [21, 26]. Here we extend these insights to explore the gamut of optical properties possible with gold semi-shells and the detailed nature of their plasmon resonances. In particular, we will show that one of the semi-shell resonances has potential for use in the design of a meta-material with anomalous magnetic permeability. 


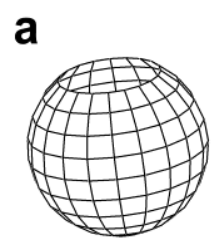

a

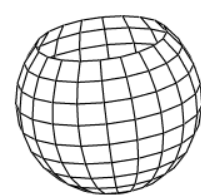

$b$

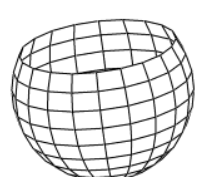

c

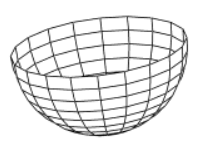

$d$

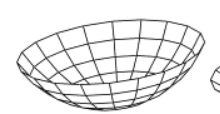

e

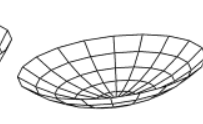

$f$

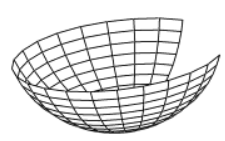

$g$

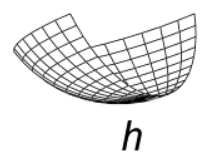

b
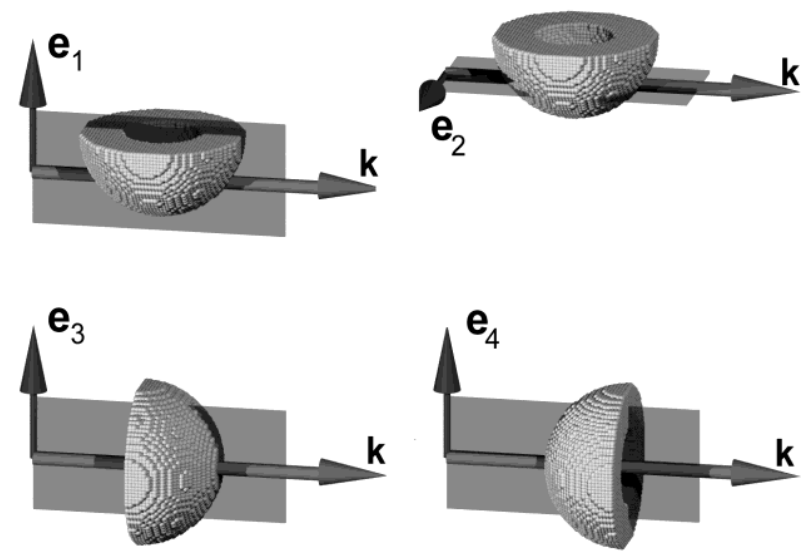

Figure 1. Semi-shells of various kinds and their orientation with respect to light. (a) The family of semi-shell shapes. Shapes $a, b$ and $c$ have been designated 'nanocups' [21], shape $d$ is a 'half-shell' [25], shapes $e$ and $f$ are 'nanocaps' [21] with rotational symmetry, and shapes $g$ and $h$ are 'nanocaps' without an axis of symmetry. (Reproduced with permission from Liu et al. [19].) The semi-shells $a$ to $f$ (but not $g$ and $h$ ) can be defined by a cut-off parameter, $\delta$, that gives their height fraction relative to a full shell $(\delta=1.0)$. For example, a half-shell has $\delta=0.5$. (b) Various orientations of light with respect to the semishells studied here. The direction of propagation of light (k) is shown as a long arrow while the electric field (e) is shown by a translucent plane and a short arrow orthogonal to $\mathbf{k}$.

\section{Methodology}

The baseline to which all shapes are referred in the present work is a full nanoshell, and we have simulated their optical properties using the Discrete Dipole Approximation code of Draine and Flatau $[27,28]$. In this scheme the nanoparticle is approximated by a three-dimensional array of dipoles and the resulting optical behaviour obtained numerically. The accuracy of this code has been extensively verified [12, 29, 30]; however it does have the twin limitations (for metallic nanoparticles at least) of slow convergence at wavelengths greater than $700 \mathrm{~nm}$, and a requirement to use a small dipole. These factors impose severe computational limitations on the simulation of particles with a volume of more than about $5 \times 10^{5} \mathrm{~nm}^{3}$ (which corresponds, for example, to the volume of a solid sphere of $100 \mathrm{~nm}$ diameter). Nevertheless, DDSCAT simulations of nanoshells closely replicate the exact analytical expressions, where available, for smaller sized particles particularly if a sufficiently fine dipole spacing is used. In previous work on rods [31] we used a dipole volume of $\sim 1 \mathrm{~nm}^{3}$. Unfortunately, the volume of 
gold in the present shapes is considerably larger, and such a small dipole volume would require an impractically large number of dipoles. In the present work we found that a dipole volume of between 5 to $10 \mathrm{~nm}^{3}$ was adequate, since it reproduced the position of the optical extinction of the analytical solution for a nanoshell [32] within reasonable computation times (see Figure S1 in Supporting Information). However, the numerical method underestimates the extinction efficiency (the ratio of ostensible optical cross-section to actual geometric cross-section, $Q_{\text {ext }}$ ) of the extinction peak, with the best result (corresponding to the $5 \mathrm{~nm}^{3}$ dipole) being a $14 \%$ under-estimate. Use of coarser dipoles exacerbates this problem and also introduces numerical instability at higher wavelengths. We also note that the $Q_{\text {ext }}$ of DDSCAT is defined with respect to an 'effective radius' of nanoparticle [28], which is not the same as the outer radius of a nanoshell.

Although the DDSCAT code does not normally output any information on the dipoles, the numerical solution for them is contained within the work space of the program. We modified DDSCAT to output this data, which consists of a 3-dimensional array of phasors. The array was then processed to yield maps and animations of the ostensible electric dipoles in the nanoparticles under various conditions.

In the present work we consider only semi-shells with rotational symmetry. Four orientations of the electric field and $k$ vectors of the light are considered in this work, figure 1(b). Besides outer diameter and shell thickness, the present semi-shells are also defined by $\delta$, the cut-off parameter, which is their fractional height compared to the full nanoshell geometry $(\delta=1)$. The effect of surface roughness was simulated by randomly placing a predetermined number of hemispherical gold blebs on an empty core, much as described in a previous publication [33]. The material of the shell in all calculations is gold and the core and surrounding medium is water. The bulk dielectric properties of gold were used. This is acceptable for shell thicknesses greater than $10 \mathrm{~nm}$, but electron confinement effects in thinner shells or smaller particles will occur, causing the real extinction peaks to broaden and lose intensity although leaving their wavelength substantially unchanged $[34,35]$. There is also an issue in DDSCAT itself, in which a somewhat inappropriate polarizability is used for surface dipoles [27], an effect obviously exacerbated in particles with the highest surface-to-volume ratios. We consider, however, that these factors have no material influence on our overall results, because their effects are restricted to some attenuation and shift in the plasmon resonances of only the thinnest of the semi-shells simulated here.

\section{Results}

In figure 2 we show the orientation and amplitude of the dipoles in a nanoshell of $100 \mathrm{~nm}$ outer diameter and $15 \mathrm{~nm}$ shell thickness at the resonant position, and for the phase angles depicted (an animation of the dipoles is available as Movie01.avi in the Supporting Information). In this case the resonance is of a simple dipolar nature and, overall, the charge simply oscillates back and forth in the plane of e, the electric field of the light. In detail, however, the resonance is longitudinal in nature at positions $\mathrm{P}$ and $\mathrm{T}$, transverse at positions $\mathrm{R}$ and $\mathrm{V}$, and of an anti-symmetric nature at the transition zones of $\mathrm{Q}, \mathrm{S}, \mathrm{U}$, and $\mathrm{X}$. The important point to note is that the dipoles at $\mathrm{P}$ and $\mathrm{T}$ point in the same direction at a given instant in time. We will designate this type of resonance as the $\alpha$ resonance in the text that follows. 
a

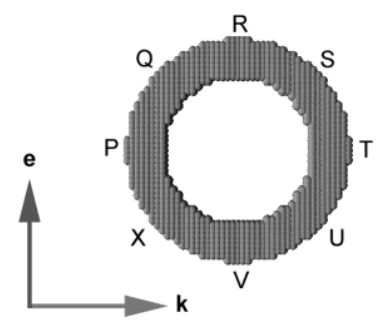

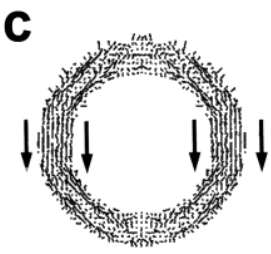
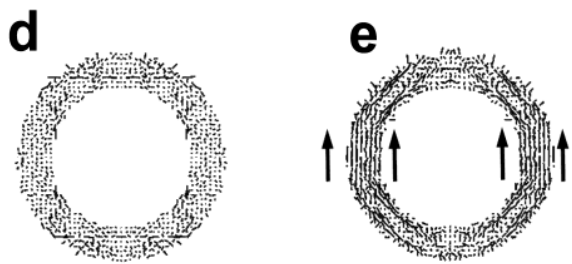

Figure 2. The $\alpha$ dipole resonance in a gold nanoshell of $100 \mathrm{~nm}$ outer diameter and $70 \mathrm{~nm}$ inner diameter. The core and surrounding medium is water. (a) Section considered for analysis of dipoles and associated electric field and propagation vectors, (b) calculated optical extinction spectrum, (c) dipole distribution and orientation at $0.194 * 2 \pi$ through cycle, (d) $0.417 * 2 \pi$, and (e) $0.667 * 2 \pi$. The overall direction in which the dipoles are pointing at $\mathrm{P}$ and $\mathrm{T}$ is shown by the arrows and each dipole is rendered as a vector with starting point anchored in the lattice grid (see Supporting Information for animations of the dipoles).

We now consider what happens when material is successively removed from the top of the nanoshell shown above. As $\delta$ is decreased below 0.85 a hole opens at the top of the semi-shell and it becomes a nano-cup $(0.85>\delta>0.5)$, then a half-shell $(\delta=0.5)$ and finally a nano-cap $(0.5>\delta>0.15)$. The series of shapes, and the average of their $\mathbf{e}_{1}$ and $\mathbf{e}_{2}$ optical extinction spectra, are shown in figure 3(a). It is clear that for $1.0>\delta>0.85$ the optical properties remain those of a nanoshell with an $\alpha$ resonance at 610 $\mathrm{nm}$, but as soon as the hole appears $(\delta \approx 0.85)$ a strong new resonance develops at $690 \mathrm{~nm}$. We will designate this the $\beta$ resonance. The nanoshell $\alpha$ resonance is generated in the semishell by only by the $\mathbf{e}_{1}$ orientation, and this weakens, blue shifts, and disappears as $\delta$ decreases. There is also a third, smallish peak at $\sim 580 \mathrm{~nm}$, which will be designated the $\gamma$ resonance. A separation of the effects due to the various orientations, figure 3(b), shows that the $\beta$ and $\gamma$ resonances are associated with the $\mathbf{e}_{2}, \mathbf{e}_{3}$ and $\mathbf{e}_{4}$ orientations, which produce identical optical extinction spectra. Although possibly counter-intuitive, it is actually the direction of the electric field that determines the optical extinction spectrum of a single nanoparticle, and the direction of the $\mathbf{k}$ vector is not important [26]. In the $\mathbf{e}_{2}, \mathbf{e}_{3}$ and $\mathbf{e}_{4}$ cases the field is directed at right angles to the axis of rotational symmetry of the semishell, while for $\mathbf{e}_{1}$ it is parallel to it. Of course, in arrays or suspensions of nanoparticles there are macroscopic geometric effects that do depend on $\mathbf{k}$, for example the extent by which the light is attenuated, and we will show later that there should be an anomalous magnetic permeability that depends on both e and k. The $Q_{\text {ext }}$ values at the various peaks can be extracted, and this is shown in figure 3(c) for the case of light with a single (appropriate) orientation.

The evolution of dipole orientations and magnitudes with time can be used again to characterize the different resonances, figure 4. The resonances at 610 and $690 \mathrm{~nm}$ are dipolar in nature, with the local dipole moment oscillating back and forth (Movie02 and Movie03 respectively in Supporting Information), but the $\alpha$ resonance (at $610 \mathrm{~nm}$ ) is along the short direction of the nanoparticle while the $\beta$ resonance (at $690 \mathrm{~nm}$ ) is perpendicular to the axis of rotational symmetry. The oscillation of the $\beta$ resonance is influenced by the presence of the gap corresponding to the mouth of the particle, which causes it to have a somewhat asymmetric nature, being far stronger on the side of the particle opposite the mouth of the semishell. This causes the $\beta$ resonance to have a circular, longitudinal aspect which 
will have the net effect of causing an oscillating current loop, the practical importance of which we will return to later. Once the mouth of the particle closes the $\beta$ resonance disappears completely because the circuit for the charge flow is then complete. It appears necessary to interrupt this path in order to have charge build-up. Next we consider the $\gamma$ resonance. The maximum in the magnitude of the dipoles (arrows, figure 4) at the 6 o'clock position leads that at the 10 and 2 o'clock positions by $\pi / 4$ radians. Furthermore, the oscillations at the 10 and 2 o'clock positions are directed in opposite directions. The phenomenon is particularly apparent if the animation is viewed. (Movie04 in Supporting Information) Therefore, this is the quadrupole resonance identified in previous work on semi-shells [21, 26] and it reaches its greatest strength in this series of shapes when $\delta=0.85$. However, the wavelengths at which the $\alpha, \beta$ and $\gamma$ resonances occur approach one another as $\delta$ is increased from 0.2 to 0.85 . For $0.85<\delta<$ 1.0 the peaks overlap so much that the individual resonances can no longer be differentiated.

Of course, it is not only $\delta$ that is important, and aspect ratio and surface roughness also play an important role. In figure 5(a) we examine the extinction spectra of a range of semi-shells of $100 \mathrm{~nm}$ outer diameter and $\delta=0.8$, but with varying shell thicknesses. The particle with the $25 \mathrm{~nm}$ thick shell behaves almost like a solid sphere, but as the shell is thinned the separation between the nanoshell-like resonance due to $\mathbf{e}_{1}$ and the new circular, longitudinal resonance due to $\mathbf{e}_{2}$ increases, while both are simultaneously red-shifted. The onset of diverse multipolar effects is also visible in the calculated spectra as evidenced by small irregularities on the $Q_{\text {ext }}$ curves. Surface roughness, if present, is also known to have a red-shifting effect $[36,37]$ and the effect is simulated in figure 5(b) for a series of shapes in which a half shell of $100 \mathrm{~nm}$ outside diameter and $20 \mathrm{~nm}$ shell thickness has been constructed from an increasing number of hemispherical gold sub-grains. In practice the surface of the semi-shells is likely to be quite rough, irrespective of whether produced by chemical or physical means (see, for example, images published elsewhere $[20,26])$. A poorly covered templating sphere can be seen to have a broad optical extinction peak due to the somewhat random dipole-dipole interactions between individual gold blebs, but as the coverage of the gold increases (which simultaneously reduces the surface roughness) the spectrum develops two clear resonances, in this case at about 550 and $670 \mathrm{~nm}$. The effect of surface roughness is to red-shift the second resonance, as expected. 

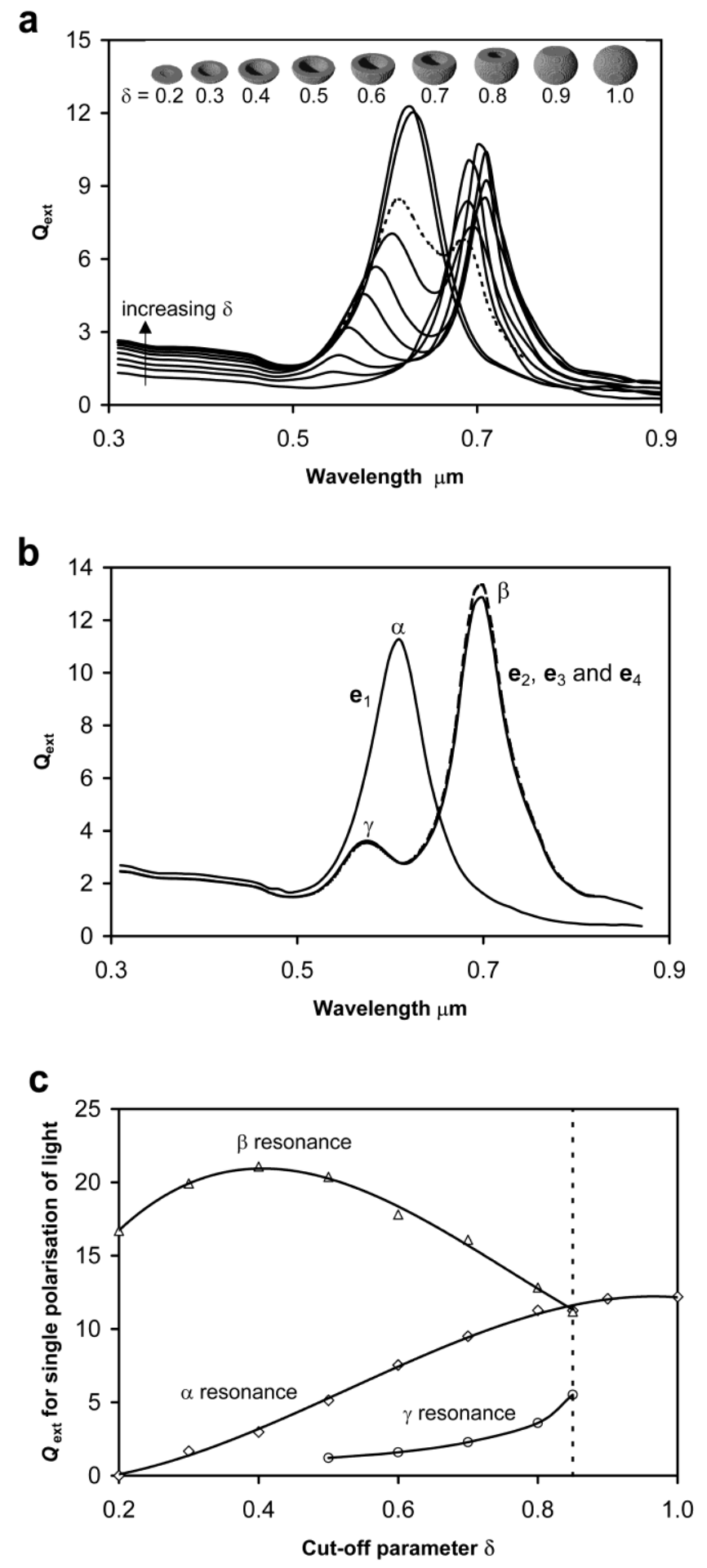

Figure 3. Conversion of a nanoshell to a semi-shell by removal of material, and the effect of this action on the calculated optical extinction spectra. (a) Average of $\mathrm{e}_{1}$ and $\mathrm{e}_{2}$ orientations for $\delta$ from 0.2 to 1.0, also showing the shapes simulated. Data for $\delta=0.85$ is shown as a dotted line. (b) Separation of effects due to orientation of light for $\delta=0.8$ (also showing the effect of orientations $\mathrm{e}_{3}$ and $\mathrm{e}_{4}$ as dashed lines). (c) Peak $Q_{\mathrm{ext}}$ values of main resonances as a function of $\delta$ for this series of semi-shells, extracted for a single but appropriate orientation of the light. The wavelength at which the three resonances occur converge near $\delta=0.85$ (dotted line) and they cannot be distinguished from one another when $0.85<\delta<1.0$. 
a
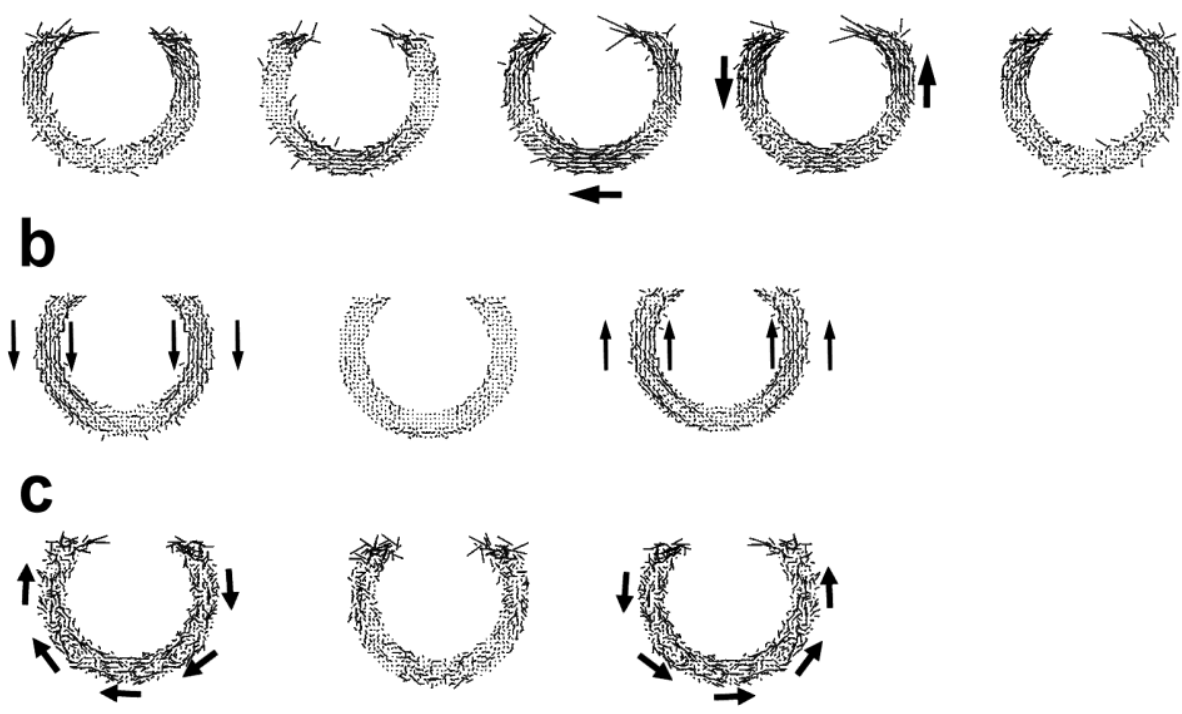

Figure 4. Nature of the dipole resonances in gold semi-shells of $100 \mathrm{~nm}$ outer diameter, $15 \mathrm{~nm}$ shell thickness, and $\delta=0.8$. Maxima in local dipole moment shown by arrows. (a) Quadrupole $\gamma$ resonance at $570 \mathrm{~nm}$, with images separated by $\theta=\pi / 4$ radians, (b) dipole $\alpha$ resonance at $610 \mathrm{~nm}$ sampled at $\theta=\pi / 2$ intervals, and (c) longitudinal $\beta$ resonance at $670 \mathrm{~nm}$ (see Supporting Information for animations of the dipoles).

\section{Discussion}

Although other precious metal nanoparticle shapes can also be tuned to absorb at varying wavelengths, semi-shells are especially interesting. First, the anisotropic nature of their optical properties can potentially be readily exploited in arrays prepared on transparent substrates by physical or chemical methods. This yields coatings of angular- and wavelength-selective properties [21,26]. Another factor is that the dipole strengths at the rim of the semi-shell are large, much larger than at the apex of a nanoshell. This will produce large electric fields in the vicinity of the mouth of the semi-shells [38], possibly rendering them useful in respect of fluorescence enhancement or Surface Enhanced Raman analyses. Finally, the diverse nature of the various plasmon resonances of nanoshells may open up possibilities for the design and synthesis of meta-materials. In particular, nanoscale current loops have been predicted shown to have interesting effects on the effective optical and magnetic properties of a composite of conducting rods in an insulating matrix [39]. This is the reason why metamaterial schemes intended for functionality in the visible or near-infrared part of the spectrum are currently generally based on rods, bars or cylinders (e.g. [40-42]), and their longitudinal and transverse plasmon resonances. 


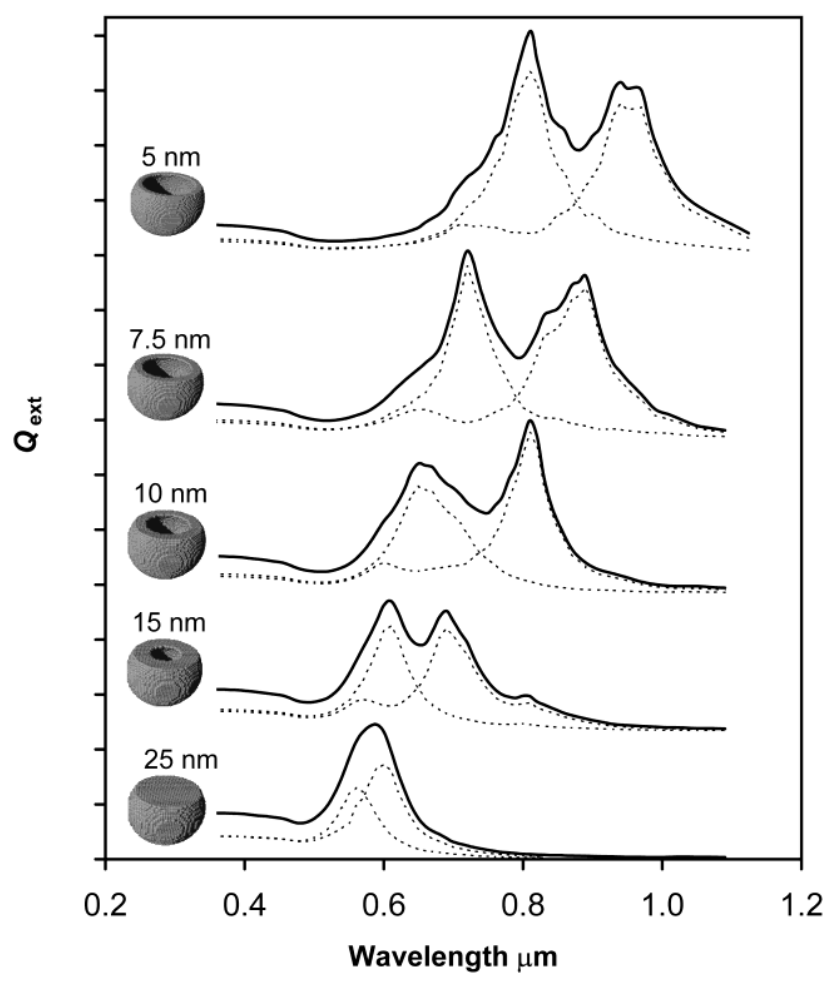

b

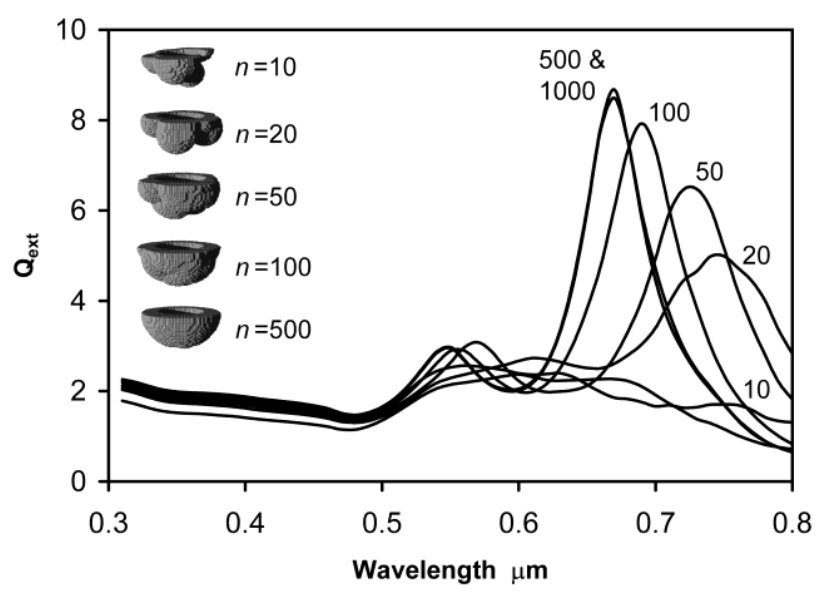

Figure 5. Effect of varying shell thickness and surface roughness on semi-shells of $100 \mathrm{~nm}$ outer diameter. (a) $\delta=0.8$, and shell thickness as indicated. In all cases the left-hand peak is generated by $\mathbf{e}_{1}$, and the right-hand peak by $\mathbf{e}_{2}, \mathbf{e}_{3}$ or $\mathbf{e}_{4}$. (b) Effect of surface roughness on the calculated optical extinction spectrum of a gold half shell. The number of blebs of gold used to generate the shapes is shown next to a depiction of each shape and alongside each spectrum. The inner diameter of the shapes is $60 \mathrm{~nm}$, and the blebs are $20 \mathrm{~nm}$ thick at their mid-points.

The plasmon resonance shown in figure 4(c) is effectively a current loop that reverses once during the passage of one wave of light of the appropriate frequency. From the right-hand rule it can be deduced that this current loop will set up a magnetic field which will also oscillate. For light of the correct wavelength and with the $\mathbf{e}_{3}$ orientation this field will add to that of the incoming light (with a small phase lag), but for the $\mathbf{e}_{4}$ orientation it will oppose it, figure 6 . For this special wavelength and for these two orientations, an array of these shapes should exhibit an enhanced apparent magnetic permeability and a reduced, perhaps even negative, apparent magnetic permeability, respectively. Is it possible to dispense with the semi-shell and achieve the same effect using a split ring? The answer is that it is not, 
because the $\mathbf{k}$ of the light must necessarily lie in the plane of the ring, a geometry that would cause extreme attenuation if the rings are deposited on the surface of a planar substrate. This limitation does not exist for semi-shells, which would normally be prepared by physical vapour deposition in the appropriate orientation on a substrate.

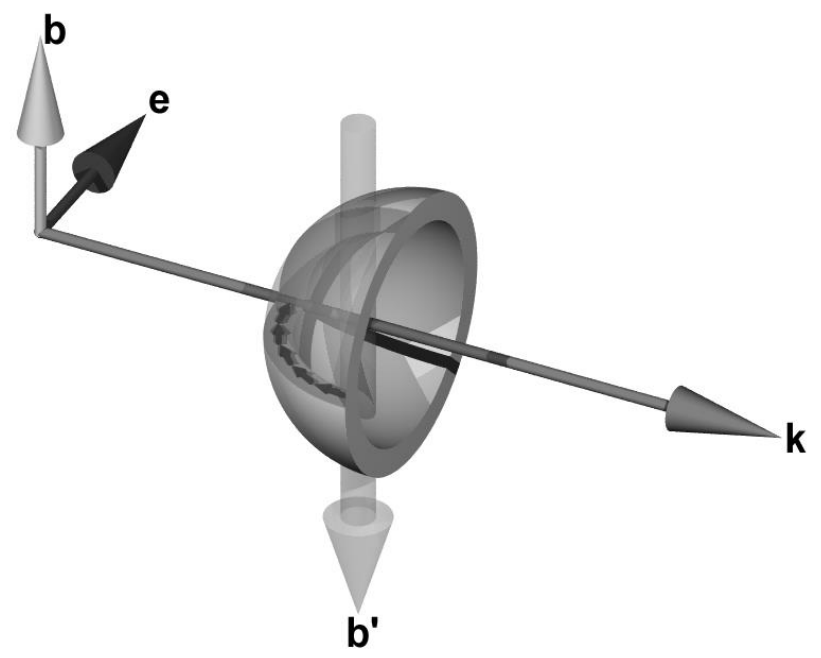

Figure 6. Gold half-shell under conditions of reduced magnetic permeability. The $\mathbf{k}$, $\mathbf{e}$ and $\mathbf{b}$ vectors of the light are shown, along with the induced current in the half-shell (small arrows inside cut-away shape), and the induced magnetic field, $\mathbf{b}^{\prime}$ (translucent downward pointing arrow).

\section{Conclusions}

Gold semi-shells exhibit additional plasmon resonances with light as a result of their reduced symmetry compared to full nanoshells. We have investigated these resonances by simulating the optical properties of family of shapes sharing an outer diameter of $100 \mathrm{~nm}$. As the semi-shell shape is produced by cutting back a nanoshell, the basic nanoshell resonance is sustained until the top of the shell is perforated. Thereafter a longitudinal and a quadrupole resonance develop, while the contribution of the nanoshell resonance declines. The longitudinal resonance is interesting because it generates an oscillating current loop. This in turn is expected to generate a net magnetic field. Depending on the orientation of the light with respect to the nanoparticle, this will either enhance or reduce the apparent magnetic permeability of the nanoparticle. We propose that arrays of these nanoparticles will provide a useful basis for a metamaterial with anomalous magnetic permeability.

Acknowledgement This research was supported by the Australian Research Council. The authors thank B. Draine and P. Flatau for use of their DDSCAT program.

Supporting Information Available. Animations of the dipole resonances. 


\section{References}

[1] Iwakoshi, A., Nanke, T. and Kobayashi, T. 2005 Gold Bull. 38(3) 107.

[2] Wagner, F. E., Haslbeck, S., Stievano, L., Calogero, S., Pankhurst, Q. A. and Martinek, P. 2000 Nature 407691.

[3] Elghanian, R., Storhoff, J. J., Mucic, R. C., Letsinger, R. L. and Mirkin, C. A. 1997 Sci. 277 1078 .

[4] Xu, X., Stevens, M. and Cortie, M. B. 2004 Chem. Mater. 16(1) 2259.

[5] Xu, X., Gibbons, T. and Cortie, M. B. 2006 Gold Bull. 39(4) 156.

[6] Aslan, K., Leonenko, Z., Lakowicz, J. R. and Geddes, C. D. 2005 J. Phys. Chem. B 109(8) 3157.

[7] Loo, C., Lin, A., Hirsch, L., Lee, M. H., Barton, J., Halas, N., West, J. and Drezek, R. 2004 Technol. in Cancer Res. \& Treatment 3(1) 33.

[8] O'Neal, D. P., Hirsch, L. R., Halas, N. J., Payne, J. D. and West, J. L. 2004 Cancer Lett 209(2) 171.

[9] Pissuwan, D., Valenzuela, S. and Cortie, M. B. 2006 Trends Biotechnol. 24(2) 62.

[10] Murphy, C. J., Sau, T. K., Gole, A. M., Orendorff, C. J., Gao, J., Gou, L., Hunyadi, S. E. and Li, T. 2005 J. Phys. Chem. B 10913857.

[11] Cortie, M. B., Xu, X. and Ford, M. J. 2006 Physical Chemistry : Chemical Physics 83520.

[12] Kelly, K. L., Coronado, E., Zhao, L. L. and Schatz, G. C. 2003 J. Phys. Chem. B 107668.

[13] Averitt, R. D., Sarkar, D. and Halas, N. J. 1997 Phys. Rev. Lett 78(22) 4217.

[14] Zhou, H. S., Honma, I., Komiyama, H. and Haus, J. W. 1994 Physical Rev. B 50(16) 12052.

[15] Neeves, A. E. and Birnboim, M. H. 1989 J. Opt. Soc. Am. B 6787.

[16] Kerker, M. and Blatchford, C. G. 1982 Physical Review B 26(8) 4052.

[17] Oldenburg, S. J., Averitt, R. D., Westcott, S. L. and Halas, N. J. 1998 Chem. Phys. Lett. 288243.

[18] Oldenburg, S. J., Jackson, J. B., Westcott, S. L. and Halas, N. J. 1999 Appl. Phys. Lett. 75(19) 2897.

[19] Liu, J., Cankurtaran, B., McCredie, G., Ford, M., Wieczorek, L. and Cortie, M. 2005 Nanotechnology 163023.

[20] Liu, J., Maaroof, A. I., Wieczorek, L. and Cortie, M. B. 2005 Adv. Mater. 17(10) 1276

[21] Charnay, C., Lee, A., Man, S., Moran, C. E., Radloff, C., Bradley, R. K. and Halas, N. J. 2003 J. Phys. Chem. B 107(30) 7327.

[22] Himmelhaus, M. and Takei, H. 2000 Sensors and Actuators, B: Chemical Sensors and Materials 6324.

[23] Takei, H. 1999 J. Vac. Sci. Technol. B 171906.

[24] Takei, H., Himmelhaus, M. and Okamoto, T. 2002 Opt. Lett. 27(5) 342.

[25] Love, J. C., Gates, B. D., Wolfe, D. B., Paul, K. E. and Whitesides, G. M. 2002 Nano Lett. 2(8)

891.

[26] Liu, J., Cankurtaran, B., Wieczorek, L., Ford, M. J. and Cortie, M. B. 2006 Adv. Func. Mater. 16(11) 1457.

[27] Draine, B. T. and Flatau, P. J. 1994 J. Opt. Soc. Am. A 11(4) 1491.

[28] Draine, B. T. and Flatau, P. J., User Guide for the Discrete Dipole Approximation Code DDSCAT 6.1, 2004, http://arxiv.org/abs/astro-ph/0309069, accessed January 2005.

[29] Brioude, A., Jiang, X. C. and Pileni, M. P. 2005 J. Phys. Chem. B 10913138.

[30] Felidj, N., Aubard, J. and Levi, G. 1999 J. of Chemical Physics 111(3) 1195.

[31] Xu, X. and Cortie, M. B. 2006 Adv. Func. Mater. 16(16) 2170.

[32] Bohren, C. F. and Huffman, D. R. 1998 Absorption and Scattering of Light by Small Particles, (New York: Wiley).

[33] Peceros, K. E., Xu, X., Bulcock, S. R. and Cortie, M. B. 2005 J. Phys. Chem. B 109(46) 21516.

[34] Alvarez, M. M., Khoury, J. T., Schaaff, T. G., Shafigullin, M. N., Vezmar, I. and Whetten, R. L. 1997 J. Phys. Chem. B 1013706.

[35] Prodan, E., Nordlander, P. and Halas, N. J. 2003 Nano Lett. 3(10) 1411. 
[36] Wang, H., Goodrich, G. P., Tam, F., Oubre, C., Nordlander, P. and Halas, N. J. 2005 J. Phys. Chem. B 109(22) 11083.

[37] Nehl, C. L., Grady, N. K., Goodrich, G. P., Tam, F., Halas, N. J. and Hafner, J. H. 2004 Nano Lett. 4(12) 2355.

[38] Cankurtaran, B., Ford, M. J. and Cortie, M. 2006 Local electromagnetic fields surrounding gold nano-cap particles, 2006 International Conference on Nanoscience and Nanotechnology Proceedings, Brisbane, Australia, 3rd-7th July, C. Jagadish, G. Q. M. Lu (eds.), p. 478.

[39] Lagarkov, A. N. and Sarychev, A. K. 1996 Physical Rev. B 53(10) 6318.

[40] Chettiar, U. K., Kildishev, A. V., Klar, T. A. and Shalaev, V. M. 2006 Optics Express 14(17) 7872.

[41] Grigorenko, A. N., Geim, A. K., Gleeson, H. F., Zhang, Y., Firsov, A. A., Khrushchev, I. Y. and Petrovic, J. 2005 Nature 438335.

[42] Zhang, S., Fan, W., Malloy, K. J., Brueck, S. R., Panoiu, N. C. and Osgood, R. M. 2005 Optics Express 134922. 


\section{Figure captions}

Figure 1. Semi-shells of various kinds and their orientation with respect to light. (a) The family of semi-shell shapes. Shapes $a, b$ and $c$ have been designated 'nanocups' [21], shape $d$ is a 'half-shell' [25], shapes $e$ and $f$ are 'nanocaps' [21] with rotational symmetry, and shapes $g$ and $h$ are 'nanocaps' without an axis of symmetry. (Reproduced with permission from Liu et al. [19].) The semi-shells $a$ to $f$ (but not $g$ and $h$ ) can be defined by a cut-off parameter, $\delta$, that gives their height fraction relative to a full shell $(\delta=1.0)$. For example, a half-shell has $\delta=0.5$. (b) Various orientations of light with respect to the semishells studied here. The direction of propagation of light $(\mathbf{k})$ is shown as a long arrow while the electric field (e) is shown by a translucent plane and a short arrow orthogonal to $\mathbf{k}$.

Figure 2. The $\alpha$ dipole resonance in a gold nanoshell of $100 \mathrm{~nm}$ outer diameter and $70 \mathrm{~nm}$ inner diameter. The core and surrounding medium is water. (a) Section considered for analysis of dipoles and associated electric field and propagation vectors, (b) calculated optical extinction spectrum, (c) dipole distribution and orientation at $0.194 * 2 \pi$ through cycle, (d) $0.417 * 2 \pi$, and (e) $0.667 * 2 \pi$. The overall direction in which the dipoles are pointing at $\mathrm{P}$ and $\mathrm{T}$ is shown by the arrows and each dipole is rendered as a vector with starting point anchored in the lattice grid (see Supporting Information for animations of the dipoles).

Figure 3. Conversion of a nanoshell to a semi-shell by removal of material, and the effect of this action on the calculated optical extinction spectra. (a) Average of $\mathrm{e}_{1}$ and $\mathrm{e}_{2}$ orientations for $\delta$ from 0.2 to 1.0, also showing the shapes simulated. Data for $\delta=0.85$ is shown as a dotted line. (b) Separation of effects due to orientation of light for $\delta=0.8$ (also showing the effect of orientations $\mathrm{e}_{3}$ and $\mathrm{e}_{4}$ as dashed lines). (c) Peak $Q_{\text {ext }}$ values of main resonances as a function of $\delta$ for this series of semi-shells, extracted for a single but appropriate orientation of the light. The wavelength at which the three resonances occur converge near $\delta=0.85$ (dotted line) and they cannot be distinguished from one another when $0.85<\delta<$ 1.0 .

Figure 4. Nature of the dipole resonances in gold semi-shells of $100 \mathrm{~nm}$ outer diameter, $15 \mathrm{~nm}$ shell thickness, and $\delta=0.8$. Maxima in local dipole moment shown by arrows. (a) Quadrupole $\gamma$ resonance at $570 \mathrm{~nm}$, with images separated by $\theta=\pi / 4$ radians, (b) dipole $\alpha$ resonance at $610 \mathrm{~nm}$ sampled at $\theta=\pi / 2$ intervals, and (c) longitudinal $\beta$ resonance at $670 \mathrm{~nm}$ (see Supporting Information for animations of the dipoles).

Figure 5. Effect of varying shell thickness and surface roughness on semi-shells of $100 \mathrm{~nm}$ outer diameter. (a) $\delta=0.8$, and shell thickness as indicated. In all cases the left-hand peak is generated by $\mathbf{e}_{1}$, and the right-hand peak by $\mathbf{e}_{2}, \mathbf{e}_{3}$ or $\mathbf{e}_{4}$. (b) Effect of surface roughness on the calculated optical extinction spectrum of a gold half shell. The number of blebs of gold used to generate the shapes is shown next to a depiction of each shape and alongside each spectrum. The inner diameter of the shapes is $60 \mathrm{~nm}$, and the blebs are $20 \mathrm{~nm}$ thick at their mid-points.

Figure 6. Gold half-shell under conditions of reduced magnetic permeability. The $\mathbf{k}$, $\mathbf{e}$ and $\mathbf{b}$ vectors of the light are shown, along with the induced current in the half-shell (small arrows inside cut-away shape), and the induced magnetic field, $\mathbf{b}^{\prime}$ (translucent downward pointing arrow). 